\title{
Use of Video Telehealth Tablets to Increase Access for Veterans Experiencing Homelessness
}

\author{
Lynn A. Garvin, PhD, MBA ${ }^{1,2}$ (D) Jiaqi Hu, MPH, MBBS 3,4 , Cindie Slightam, $M P H^{3}$, \\ D. Keith Mclnnes, SCD, $M S^{2,5}$, and Donna M. Zulman, MD, $M S^{3,4}$
}

'VA Center for Healthcare Organization and Implementation Research (CHOIR), VA Boston Healthcare System, 150 S. Huntington Avenue, Bldg 9, Rm 225, Boston, MA, USA; '2Department of Health Law, Policy and Management, Boston University School of Public Health, Boston, MA, USA; ${ }^{3}$ VA Center for Innovation to Implementation (Ci2i), VA Palo Alto Health Care System, Menlo Park, CA, USA; ${ }^{4}$ ivision of Primary Care and Population Health, Stanford University School of Medicine, Stanford, CA, USA; ${ }^{5}$ VA Center for Healthcare Organization and Implementation Research (CHOIR), VA Bedford Healthcare System, Bedford, MA, USA.

BACKGROUND: Veterans experiencing homelessness face substantial barriers to accessing health and social services. In 2016, the Veterans Affairs (VA) healthcare system launched a unique program to distribute videoenabled tablets to Veterans with access barriers.

OBJECTIVE: Evaluate the use of VA-issued video telehealth tablets among Veterans experiencing homelessness in the VA system.

DESIGN: Guided by the RE-AIM framework, we first evaluated the adoption of tablets among Veterans experiencing homelessness and housed Veterans. We then analyzed health record and tablet utilization data to compare characteristics of both subpopulations, and used multivariable logistic regression to identify factors associated with tablet use among Veterans experiencing homelessness.

PATIENTS: In total, 12,148 VA patients receiving tablets between October 2017 and March 2019, focusing on the 1470 VA Veterans experiencing homelessness receiving tablets (12.1\%).

MAIN MEASURES: Tablet use within 6 months of receipt for mental health, primary or specialty care.

KEY RESULTS: Nearly half (45.9\%) of Veterans experiencing homelessness who received a tablet had a video visit within 6 months of receipt, most frequently for telemental health. Tablet use was more common among Veterans experiencing homelessness who were younger $(\mathrm{AOR}=2.77 ; P<.001) ;$ middle-aged $(\mathrm{AOR}=2.28 ; P$ $<.001)$; in rural settings (AOR $=1.46 ; P=.005)$; and those with post-traumatic stress disorder $(\mathrm{AOR}=1.64 ; P<.001)$, and less common among those who were Black $(\mathrm{AOR}=$ 0.43; $P<.001$ ) and those with a substance use disorder (AOR $=0.59 ; P<.001)$ or persistent housing instability $(\mathrm{AOR}=0.75 ; P=.023)$.

CONCLUSIONS: Telehealth care and connection for vulnerable populations are particularly salient during the COVID-19 pandemic but also beyond. VA's distribution

D. Keith McInnes and Donna M. Zulman contributed equally to this manuscript.

Prior Presentations

Garvin LA, Hu J, Slightam C, McInnes DK, Zulman DM. Virtual Outreach: Enhancing Access for Homeless Veterans through Video Telehealth Tablets. Oral presentation at the 2020 Virtual Annual Research Meeting of AcademyHealth on August 6, 2020.

Received July 5, 2020

Accepted May 3, 2021

Published online May 23, 2021 of video telehealth tablets offers healthcare access to Veterans experiencing homelessness; however, barriers remain for subpopulations. Tailored training and support for these patients may be needed to optimize telehealth tablet use and effectiveness.

KEY WORDS: homelessness; Veterans; telemedicine; health services accessibility; mental health.

J Gen Intern Med 36(8):2274-82

DOI: $10.1007 / \mathrm{s} 11606-021-06900-8$

(c) This is a U.S. government work and not under copyright protection in the U.S.; foreign copyright protection may apply 2021

$\mathrm{T}$ he US Department of Veterans Affairs (VA) is a leader in clinical video telehealth to increase Veterans' access to high-quality care. ${ }^{1}$ In 2018 alone, VA healthcare systems provided more than 2.29 million telehealth episodes of video telehealth care to $12 \%$ of eligible Veterans. ${ }^{2}$ The VA Video Connect (VVC) mobile application allows Veterans to securely stream live video sessions with their healthcare teams on the device of their choice.

Studies have shown that video telehealth can offer effective delivery of mental healthcare, ${ }^{3-5}$ primary care, ${ }^{6,7}$ and specialty ambulatory care. ${ }^{8-10}$ Patient populations who face sociodemographic and clinical challenges (e.g., travel distance to care for rural patients) can benefit from video telehealth. ${ }^{11-}$ 16 Other vulnerable populations that have been shown to benefit from video telehealth include older adults, ${ }^{17-21}$ African American and Hispanic adults, ${ }^{22-25}$ and Native and Alaskan American adults; ${ }^{26,27}$ and patients with mental health conditions such as anxiety and depression ${ }^{28}$ or anxiety and alcohol use disorder, ${ }^{29,30}$ post-traumatic stress disorder (PTSD), ${ }^{31,32}$ substance use disorder (SUD) $;^{33-35}$ or challenges with medication adherence. $^{36}$

One VA patient population that could potentially benefit from virtual care is Veterans experiencing homelessness, representing $8 \%(n=37,085)$ of all US homeless adults. This population is a VA priority in part because it is characterized by elevated mortality due to high rates of suicide and fatal overdoses. $^{37,38}$ Veterans experiencing homelessness 
encounter health- and travel-related access barriers, while stigma may interfere with their willingness to seek care. Video telehealth could overcome some of these challenges, offering a mechanism for improved access to critical clinical services in this population. ${ }^{39,40}$

Little is known about video telehealth use among Veterans experiencing homelessness and how this technology influences their access to care. In 2016, the VA began the largest known program to distribute video telehealth tablets to Veterans facing access barriers. The tablets come with data plans and Wi-Fi connectivity. ${ }^{41}$ Between October 2017 and March 2019, the VA distributed 12,148 tablets to accesschallenged Veterans. Tablets can be used for any clinical care that does not require physical contact, including mental health therapy and medication management, primary care, palliative care, and selective specialty and rehabilitation care. ${ }^{42}$

Previous evaluations have shown that the tablet distribution program successfully reached patients with clinical or social barriers and generated cost savings for such patients. ${ }^{43,44}$ In this study, we sought to examine variation in sociodemographic and clinical characteristics with tablet recipients stratified by housed vs. homeless status, and by tablet users vs. non-users among Veterans experiencing homelessness, and factors associated with their tablet use. Our findings may contribute to an understanding of how video telehealth tablets and other devices can substitute for in-person healthcare encounters in the context of the COVID-19 pandemic and beyond.

\section{METHODS}

\section{Tablet Distribution Evaluation}

This paper uses the RE-AIM framework to focus on the construct of adoption, "the level of patients' acceptance, use of, satisfaction with, and willingness to recommend to others," in this case, rates and characteristics of tablet use. ${ }^{45,46}$ The tablets were purchased by VA's Office of Rural Health (ORH) from BL Healthcare, preconfigured to be compatible with VA Office of Information and Technology requirements including encryption. Eligibility criteria included enrolled and active VA patient, not having their own device or data plan, able to operate the technology (or have a caregiver assist), and unable to access in-person VA care. Eligible patients were referred by VA providers. Patient training involved VA representatives calling tablet recipients to guide them through initial system set-up. Providers received user training on Cisco Jabber video technology to connect with patients. ${ }^{43,44}$ Homeless tablet recipients who had at least one video visit in 6 months after receipt were considered "tablet users."

This evaluation was conducted in partnership with the VA's Office of Connected Care and the Virtual Access Quality Enhancement Research Initiative at VA Palo Alto, and was designated as non-research quality improvement by the
Research and Development Committee of the VA Boston Healthcare System.

\section{Data Sources}

Data were drawn from VA's Denver Acquisitions and Logistics Center (tablet shipment information) and VA's national Central Data Warehouse (patient sociodemographic and clinical characteristics, in-person, and video clinical encounters).

\section{Measures}

Patient sociodemographics and clinical characteristics included age, sex, race/ethnicity, marital status, and rural/urban status. Rural and urban status was determined by ORH following the Rural-Urban Community Areas (RUCA) system used by the US Census Bureau. ${ }^{47}$ Patient characteristics also included VA Enrollment Priority Group which incorporates a Veteran's military service history, service-connected disability, income, Medicaid qualification, and receipt of other VA benefits. ${ }^{48}$ Priority Group 1 represents Veterans with serviceconnected disabilities; Priority Group 5 represents Veterans with low income.

Clinical diagnoses spanned 28 chronic physical health conditions and 4 mental health conditions - depression, PTSD, SUD, and serious mental illness (SMI) - and were identified using outpatient visits from the 12 months prior to tablet receipt. Clinical encounters included the type of clinic where tablets were used during the 6 months after tablet receipt: primary care, mental healthcare, specialty or other care. Indication of high risk for suicide was obtained from a VA clinical reminder from the year prior to tablet receipt. We included any in-person utilization of outpatient care and the number of inperson outpatient visits for mental health, primary care, and specialty care within 6 months of tablet receipt.

The sample included Veterans who had an indication of homelessness and had received a tablet. Homelessness was identified through the use of US Centers for Disease Control and Prevention diagnosis codes and VA Decision Support System (DSS) Identifiers. ${ }^{49,50}$ (Table 1 provides code descriptions and classification). Our measure of homelessness 6 months after tablet receipt was based on these same codes. Adoption (tablet use) was determined by a Veteran having a documented outpatient clinical video encounter within 6 months of tablet receipt (DSS code 179, for Clinical Video Telehealth into the Home). Similar adoption measures have been used in studies of patient-facing technologies such as secure messaging, telehealth, and video telehealth. ${ }^{6} \mathrm{We}$ assessed whether recipients received more than one device.

\section{Data Analyses}

Our analyses addressed five objectives: First, we examined tablet distribution among Veterans by housing status (housed vs. homeless). We calculated the proportion of Veterans experiencing homelessness among total tablet recipients. We 
Table 1 Centers for Disease Control and Prevention (CDC) Diagnosis Codes and VA Decision Support System (DSS) Identifiers Used to Determine Homelessness Among Veterans

Classification of a Veteran experiencing homelessness

For purposes of our study, a Veteran was classified as experiencing homelessness if there were one CDC International Classification of Disease ICD-9/ICD-10 code, ${ }^{49}$ specifically V60.0 (lack of housing) or Z59.0 (homelessness); or one VA DSS Identifier" (VA's 3-digit "stop code" reflecting type of outpatient care) related to the utilization of VA homelessness services $(504,507,508,511,522,528,529,530,555,556$, and 590) during the 12 months prior to tablet distribution.

CDC ICD Codes

ICD-9 Code V60.0 (lack of housing)

ICD-10 Code Z59.0 (homelessness)

VA DSS Identifiers

VA Clinic Contact Points (called “stop codes"):

- 504 Veterans receiving services from Grant and Per Diem Program Office - Group Assistance

- 507 homeless or at-risk Veterans or family members receiving services from VA clinical staff of Housing and Urban Development - VA Supported

Housing (HUD-VASH) Programs - Group Assistance

- 511 homeless Veterans or family members receiving services from Grant and Per Diem Program Office - Individual Assistance

- 522 or 530 homeless Veteran or family members receiving services from Department of Housing and Urban Development VA Shared Housing

Program [HUD-VASH])] - Individual Assistance

- $508 \mathrm{HCHV} / \mathrm{HCMI}$ Group [VA Health Care for Homeless Veterans/Homeless Chronically Ill]

- 528 telephone services/homeless mentally ill [HMI]

- 529 Health Care for Homeless Veterans (HCHV) services - restricted to programs approved by the Northeast Program Evaluation Center (NEPEC)

- 555 homeless Veteran Community Employment Services - Individual Assistance

- 556 homeless Veteran Community Employment Services - Group Assistance

- 590 community outreach to homeless Veterans

used chi-square tests to examine the differences in demographic, social, and clinical characteristics between housed and homeless tablet recipients, then compared on urban vs. rural location among Veterans experiencing homelessness. We also examined healthcare utilization by tablet recipients, specifically the proportion of video versus in-person visits. Second, using chi-square tests, we compared tablet users versus nonusers, initially among all Veterans experiencing homelessness, and for our third objective, we further stratified by urban and rural location. Fourth, we compared VA tablet utilization (completed video visits) in terms of proportion of each of 3 types of care (mental health, primary care, and specialty or other care) by housing status, and then further stratified by urban vs. rural location. Finally, we evaluated characteristics associated with tablet use through multivariable logistic regression. All bivariate analyses and regressions used a $P$-value $\leq 0.05$ as the cutoff for significance. We used complete case analysis and missing values were noted in the descriptive tables.

\section{RESULTS}

\section{Tablet Recipient Characteristics}

From October 2017 to March 2019, 12,148 Veterans from 70 VA facilities across the USA received a tablet. Of these, 474 (3.9\%) Veterans received more than one tablet. Veterans experiencing homelessness represented $12.1 \%(N=1470)$ of all tablet recipients; homeless and housed tablet recipients varied across many sociodemographic and clinical characteristics, and in-person healthcare utilization (Table 2).

\section{Tablet User Characteristics and Utilization Patterns}

Nearly half $(45.9 \%, N=675)$ of homeless recipients had used the tablet ("tablet users") for a video visit within 6 months of receipt (Table 3). In bivariate analyses, homeless tablet users were more likely than non-users to be younger (35.7\% vs. $23.9 \%$ in the $18-44$ age range), married ( $24.7 \%$ vs. $17.5 \%)$, White $(60.7 \%$ vs. $43.6 \%)$, residing in a rural location (34.9\% vs. $21.2 \%$ ), and required to drive $\geq 60 \mathrm{~min}$ to a VA facility (33.6\% vs. $21.5 \%$ ) (all results reported have $P$-values of $<0.001$ unless otherwise specified). Homeless tablet users were more likely to be in VA Priority Group 1 indicating a service-connected disability (37.8\% vs. $30.0 \% ; P=.002)$ and to have PTSD (57.9\% vs. $44.0 \%$ ). Homeless tablet users were less likely to be middle-aged $(57.5 \%$ vs. $62.5 \%)$ or older ( $6.8 \%$ vs. $13.6 \%$ ), to be Black ( $26.2 \%$ vs. $46.3 \%$ ), or to be homeless 6 months after tablet receipt $(56.1 \%$ vs. $66.4 \%)$. They were also less likely to have 3 or more chronic conditions $(48.7 \%$ vs. $56.7 \% ; P=.006)$ or to have SUD $(47.6 \%$ vs. $58.2 \%)$.

Characteristics associated with tablet use differed across urban and rural Veterans experiencing homelessness (Table 4). Among these, rural tablet users (compared to rural non-users) were more likely to be younger ( $38.4 \%$ vs. $29.1 \%$ ) and no longer homeless 6 months after tablet receipt (51.3\% vs. $41.2 \% ; P=.047$ ), were less likely to have $\geq 3$ chronic conditions (47.0 vs. $62.4 \% ; P=.008)$ or SUD diagnoses ( $48.7 \%$ vs. $60.2 \% ; P=.014$ ), but more likely to have PTSD (58.6\% vs. $47.3 \% ; P=.025)$. Urban homeless tablet users (compared to urban non-users) were more likely to be married (22.7\% vs. $15.5 \% ; P=.004)$ and either White $(53.9 \%$ vs. $35.1 \%)$ or Hispanic $(6.8 \%$ vs. $4.7 \%)$. Urban homeless tablet users were also more likely to be in Priority Group 1 indicating a service-connected disability (38.4\% vs. $29.2 \% ; P=.005)$.

Table 5 shows that telehealth utilization for different types of care differed by housing status. Veterans experiencing homelessness were more likely to use video visits for mental health $(88.0 \%$ vs. $72.1 \%)$, but less likely to use them for primary care $(5.0 \%$ vs. $9.4 \%)$ and specialty or other care (12.0\% vs. $23.6 \%)$. On average, Veterans experiencing homelessness had similar rates of mental health video visits as 
Table 2 Characteristics and Bivariate Comparisons (Unadjusted) of Veteran Telehealth Tablet Recipients

\begin{tabular}{|c|c|c|c|c|c|c|}
\hline & $\begin{array}{l}\text { All housed } \\
N=10,678(\%)\end{array}$ & $\begin{array}{l}\text { All homeless } \\
N=1470(\%)\end{array}$ & $P$-value* & $\begin{array}{l}\text { Urban } \\
\text { homeless } \\
N=\mathbf{1 , 0 4 8}(\%)\end{array}$ & $\begin{array}{l}\text { Rural } \\
\text { homeless } \\
N=397(\%)\end{array}$ & $P$-value* \\
\hline \multicolumn{3}{|l|}{ Age } & \multicolumn{3}{|l|}{$<0.001$} & \multirow[t]{4}{*}{0.010} \\
\hline $18-44$ & $2770(25.9)$ & $431(29.3)$ & & $282(26.9)$ & $137(34.5)$ & \\
\hline $45-64$ & $4092(38.3)$ & $885(60.2)$ & & $657(62.7)$ & $216(54.4)$ & \\
\hline $65+$ & $3816(35.7)$ & $154(10.5)$ & & $109(10.4)$ & $44(11.1)$ & \\
\hline \multicolumn{3}{|l|}{ Gender } & \multicolumn{3}{|l|}{0.022} & \multirow[t]{3}{*}{0.041} \\
\hline Female & 2088 (19.6) & $325(22.1)$ & & $245(23.4)$ & $73(18.4)$ & \\
\hline Male & $8587(80.4)$ & $1145(77.9)$ & & 803 (76.6) & $324(81.6)$ & \\
\hline \multicolumn{3}{|l|}{ Marital status } & \multicolumn{3}{|l|}{$<0.001$} & \multirow[t]{3}{*}{$<0.001$} \\
\hline Other & $4923(47.0)$ & $1141(79.2)$ & & $833(81.5)$ & $286(72.6)$ & \\
\hline Married & $5561(53.0)$ & $300(20.8)$ & & $189(18.5)$ & $108(27.4)$ & \\
\hline \multicolumn{3}{|l|}{ Race/ethnicity } & \multicolumn{3}{|l|}{$<0.001$} & \multirow[t]{5}{*}{$<0.001$} \\
\hline White, non-Hispanic & $6772(65.8)$ & $732(51.4)$ & & $432(42.8)$ & $290(74.0)$ & \\
\hline Black, non-Hispanic & $2177(21.2)$ & $528(37.1)$ & & $470(46.6)$ & $50(12.8)$ & \\
\hline Hispanic & $532(5.2)$ & $69(4.8)$ & & $56(5.6)$ & $12(3.1)$ & \\
\hline Other & $809(7.9)$ & $94(6.6)$ & & $51(5.1)$ & $40(10.3)$ & \\
\hline \multicolumn{3}{|l|}{ Rural/urban location } & \multicolumn{3}{|l|}{$<0.001$} & \multirow[t]{3}{*}{-} \\
\hline Rural & $4941(46.8)$ & $397(27.5)$ & & - & - & \\
\hline Urban & $5606(53.2)$ & $1048(72.5)$ & & - & - & \\
\hline \multicolumn{3}{|l|}{ Homeless after 6 months } & \multicolumn{3}{|l|}{$<0.001$} & \multirow[t]{3}{*}{$<0.001$} \\
\hline No & $10,467(98.0)$ & $563(38.3)$ & & $366(34.9)$ & $187(47.1)$ & \\
\hline Yes & $211(2.0)$ & $907(61.7)$ & & $682(65.1)$ & $210(52.9)$ & \\
\hline \multicolumn{3}{|l|}{ Enrollment priority group } & \multicolumn{3}{|l|}{$<0.001$} & \multirow[t]{4}{*}{0.510} \\
\hline Group 1: service-connected disabilities & $6273(59.3)$ & $488(33.6)$ & & $345(33.0)$ & $143(36.2)$ & \\
\hline Group 5: financial insecurity & $1561(14.8)$ & $558(38.4)$ & & $404(38.7)$ & $143(36.2)$ & \\
\hline Other than Groups 1 and 5 & $2742(25.9)$ & $408(28.1)$ & & $295(28.3)$ & $109(27.9)$ & \\
\hline \multicolumn{3}{|l|}{ Chronic conditions (from list of 28) } & \multicolumn{3}{|l|}{0.025} & 0.560 \\
\hline Zero & $1064(10.0)$ & $154(10.5)$ & & $97(9.3)$ & $44(11.1)$ & \\
\hline 1 to 2 & $3553(33.3)$ & $536(36.5)$ & & $387(36.9)$ & $134(34.11)$ & \\
\hline 3 or more & $6061(56.8)$ & $780(53.1)$ & & $564(53.8)$ & $212(53.4)$ & \\
\hline Any $\mathrm{MH}$ diagnoses & $8351(78.2)$ & $1313(89.3)$ & $<0.001$ & $931(88.8)$ & $365(91.9)$ & 0.083 \\
\hline Serious mental illness & $1107(10.4)$ & $321(21.8)$ & $<0.001$ & $219(20.9)$ & $99(24.9)$ & 0.098 \\
\hline Post-traumatic stress disorder & $5290(49.5)$ & $741(50.4)$ & 0.530 & $516(49.2)$ & $214(53.9)$ & 0.110 \\
\hline Substance use disorder & $2145(20.1)$ & $784(53.3)$ & $<0.001$ & $561(53.5)$ & $214(53.9)$ & 0.900 \\
\hline Depression & $6067(56.8)$ & $1032(70.2)$ & $<0.001$ & $737(70.3)$ & $285(71.8)$ & 0.590 \\
\hline Suicide risk flag & & & $<0.001$ & & & 0.029 \\
\hline No & $10,410(97.5)$ & $1340(91.2)$ & & $965(92.1)$ & $351(88.4)$ & \\
\hline Yes & $268(2.5)$ & $130(8.8)$ & & $83(7.9)$ & $46(11.6)$ & \\
\hline Utilization of in-person care** (unit is $v$ & & & & & & \\
\hline Any mental health & $7033(67.1)$ & $1358(93.7)$ & $<0.001$ & $965(93.2)$ & $372(94.7)$ & 0.33 \\
\hline Mental health visit, mean (SD) & $4.7(10.1)$ & $20.0(26.8)$ & $<0.001$ & $20.6(26.0)$ & $18.9(29.4)$ & 0.32 \\
\hline Any primary care & $8639(82.4)$ & $1174(81.0)$ & 0.19 & $856(82.7)$ & $305(77.6)$ & 0.027 \\
\hline Primary care visit, mean (SD) & $3.5(4.1)$ & $3.4(4.0)$ & 0.58 & $3.5(4.0)$ & $3.2(3.8)$ & 0.15 \\
\hline Any specialty care & $9643(91.9)$ & $1313(90.6)$ & 0.071 & $955(92.3)$ & $341(86.8)$ & 0.001 \\
\hline Specialty care visit, mean (SD) & $14.4(19.7)$ & $13.5(17.5)$ & 0.11 & $14.5(18.6)$ & $11.3(14.2)$ & 0. \\
\hline
\end{tabular}

For housed Veterans and Veterans experiencing homelessness: missing values exist in marital status (223), racelethnicity (435), enrollment priority group (118), geographic location (156). For urban and rural homeless tablet recipients: missing values exist in marital status (29), racelethnicity (44), enrollment priority group (6). *All P-values for continuous variables derive from t-tests and P-values for categorical variables derive from chi-square tests. **Six (6) months after tablet receipt. ${ }^{\#}$ Unlike other variables in this table for which the unit is individual Veteran, for this variable, the unit is healthcare visits

housed Veterans, but fewer primary care and specialty care visits. Rural Veterans experiencing homelessness were more likely to use video visits for mental healthcare $(94.8 \%$ vs. $84.1 \%$ ) while urban counterparts were more likely to use video visits for primary care $(6.9 \%$ vs. $1.7 \% ; P=.004)$. No difference was observed between rural and urban Veterans regarding mean mental health visits.

\section{Factors Predicting Tablet Use}

In multivariable analyses (Table 6), Veterans experiencing homelessness were more likely to have used their tablets if they were either younger $(\mathrm{AOR}=2.77,95 \% \mathrm{CI}=1.76,4.35)$ or middle-aged $(\mathrm{AOR}=2.28,95 \% \mathrm{CI}=1.52,3.43)$, and resided in a rural location $(\mathrm{AOR}=1.46,95 \% \mathrm{CI}=1.12$,
1.90), while they were less likely to have video visits if they were Black (AOR $=0.43,95 \% \mathrm{CI}=0.34,0.56$ ) or if they experienced persistent homelessness 6 months after tablet receipt $(\mathrm{AOR}=0.75, \mathrm{CI}=95 \% 0.59,0.96)(P=0.023)$. Having PTSD was associated with greater odds of tablet use $(\mathrm{AOR}=1.64,95 \% \mathrm{CI}=1.27,2.12)$, while having SUD was associated with lower odds of tablet use (AOR $=0.59,95 \% \mathrm{CI}$ $=0.46,0.76$ ).

There were few differences in regression results in tablet use when stratified by urban/rural status. The exception is that among urban Veterans experiencing homelessness, Blacks, compared to Whites, were less likely to have video visits $(\mathrm{AOR}=0.38 ; 95 \% \mathrm{CI}=0.29,0.51)$, and urban Veterans experiencing homelessness with PTSD were more likely to have video visits than those without PTSD $(\mathrm{AOR}=1.69 ; 95 \%$ 
Table 3 Characteristics and Bivariate Comparisons (Unadjusted) of Telehealth Tablet Non-users vs. Users Among Veterans Experiencing Homelessness

\begin{tabular}{|c|c|c|c|}
\hline & $\begin{array}{l}\text { All homeless } \\
\text { tablet non- } \\
\text { users } \\
N=795(\%)\end{array}$ & $\begin{array}{l}\text { All homeless } \\
\text { tablet users } \\
N=675(\%)\end{array}$ & $\begin{array}{l}P \text { - } \\
\text { value* }\end{array}$ \\
\hline Age & & $241(35.7)$ & $<0.001$ \\
\hline $18-44$ & $190(23.9)$ & $241(35.7)$ & \\
\hline $45-64$ & $497(62.5)$ & $388(57.5)$ & \\
\hline $65+$ & $108(13.6)$ & $46(6.8)$ & \\
\hline Gender & & & 0.220 \\
\hline Female & $166(20.9)$ & $159(23.6)$ & \\
\hline Male & $629(79.1)$ & $516(76.4)$ & \\
\hline Marital status & & & $<0.001$ \\
\hline Other & $641(82.5)$ & $500(75.3)$ & \\
\hline Married & $136(17.5)$ & $164(24.7)$ & \\
\hline Race/ethnicity & & & $<0.001$ \\
\hline White, non-Hispanic & $336(43.6)$ & $396(60.7)$ & \\
\hline Black, non-Hispanic & 357 (46.3) & $171(26.2)$ & \\
\hline Hispanic & $36(4.7)$ & $33(5.1)$ & \\
\hline Other & $42(5.4)$ & $52(8.0)$ & \\
\hline Rural/urban location & & & $<0.001$ \\
\hline Rural & $165(21.2)$ & $232(34.9)$ & \\
\hline Urban & $615(78.8)$ & $431(65.1)$ & \\
\hline $\begin{array}{l}\text { Homelessness after } 6 \\
\text { months }\end{array}$ & & & $<0.001$ \\
\hline No & $267(33.6)$ & $296(43.9)$ & \\
\hline Yes & $528(66.4)$ & $379(56.1)$ & \\
\hline Priority group & & & 0.002 \\
\hline $\begin{array}{l}\text { Group 1: service- } \\
\text { connected disabilities }\end{array}$ & $235(30.0)$ & $253(37.8)$ & \\
\hline $\begin{array}{l}\text { Group 5: financial } \\
\text { insecurity }\end{array}$ & $329(42.0)$ & $229(34.2)$ & \\
\hline $\begin{array}{l}\text { Other than Groups } 1 \\
\text { and } 5\end{array}$ & $220(28.1)$ & $188(28.1)$ & \\
\hline $\begin{array}{l}\text { Chronic conditions } \\
\text { (from list of } 28 \text { ) }\end{array}$ & & & 0.006 \\
\hline Zero & $72(9.1)$ & $82(12.1)$ & \\
\hline 1 to 2 & $272(34.2)$ & $264(39.1)$ & \\
\hline 3 or more & $451(56.7)$ & $329(48.7)$ & \\
\hline MH diagnoses & $691(86.9)$ & $622(92.1)$ & 0.001 \\
\hline $\begin{array}{l}\text { Serious mental } \\
\text { illness }\end{array}$ & $162(20.4)$ & 159 (23.6) & 0.140 \\
\hline $\begin{array}{l}\text { Post-traumatic stress } \\
\text { disorder }\end{array}$ & $350(44.0)$ & $391(57.9)$ & $<0.001$ \\
\hline $\begin{array}{l}\text { Substance use } \\
\text { disorder }\end{array}$ & $463(58.2)$ & $321(47.6)$ & $<0.001$ \\
\hline Depression & $554(69.7)$ & $478(70.8)$ & 0.640 \\
\hline Suicide risk flag & & & 0.900 \\
\hline No & $724(91.1)$ & $616(91.3)$ & \\
\hline Yes & $71(8.9)$ & $59(8.9)$ & \\
\hline
\end{tabular}

*All P-values derive from chi-square tests comparing tablet users and non-users

$\mathrm{CI}=1.25,2.29)$. In contrast, among rural Veterans experiencing homelessness, there was no variation in tablet use for video visits by race or PTSD diagnosis.

\section{DISCUSSION}

The VA's recent tablet distribution initiative represents the largest nationwide program to provide video-enabled tablets to patients with access barriers. While this offers many patients the technology to participate in video telehealth visits, our findings suggest that a digital divide persists, where homeless recipients are less likely to use the tablets compared with housed counterparts. The low rate $(<4 \%)$ of Veterans receiving a second tablet suggests that equipment loss/ replacement was not a substantial factor in explaining our findings.

Furthermore, among homeless recipients, a number of factors were associated with lower tablet use, including older age, Black race, urban location, and a substance use disorder. These factors are discussed in the paragraphs below. Findings suggest a need for targeted interventions to support patients experiencing homelessness and might benefit from telemedicine.

Older age may be associated with lower tablet use due to increased barriers to technology use, including physical, cognitive, and motivational challenges. ${ }^{51}$ Additionally, the combination of aging with mental health conditions, such as PTSD, can make mental and physical health symptoms (e.g., return of traumatic memories) more inhibitive to trying novel technologies. ${ }^{52}$ The experience of homelessness is associated with more rapid physiological aging, suggesting these barriers may be even more pronounced in this population.

Older individuals' adoption of technology may also relate to expectations of in-person social contact. Thus, new digital healthcare communications, such as video visits, may be more appealing as supplements, not substitutes, for in-person care. ${ }^{53} \mathrm{~A}$ study of older Veterans suggests they would benefit from simplified computer application designs and digital literacy training to increase comfort, confidence, and willingness to use. ${ }^{54}$

People of color frequently face disparities in access to healthcare. In our study, Black Veterans experiencing homelessness represented $37 \%$ of tablet recipients, but only $26 \%$ of tablet users. Our analyses are consistent with recent research showing that Black Veterans, compared to White Veterans, are less likely to use VA's My HealtheVet patient portal and clinical video telehealth. ${ }^{55}$ Implicit bias on the part of healthcare workers and structural racism in the healthcare system may also contribute to the disparities seen. ${ }^{56,57}$ Additionally, as a result, Black patients' lower levels of trust in health professionals, compared to White patients, could potentially dampen interest in sharing personal health information through VA video visits. Recent work suggests that cultural tailoring of recruitment materials and outreach approaches can generate more interest in virtual healthcare among specific racial and/or ethnic groups. ${ }^{22,58}$

Our finding that SUD was associated with reduced likelihood of video visits is consistent with other research indicating that patients actively using substances can have difficulty keeping video appointments and concentrating during visits, and express lower interest in interacting with healthcare providers by video. ${ }^{59}$ Yet telehealth holds promise for Veterans with SUD. A systematic review examined the use of mobile health interventions for the prevention of alcohol and substance use, finding that such interventions were feasible and effective. ${ }^{60}$ A recent study of VA tablets found that many Veterans with SUD prefer video visits to in-person visits. ${ }^{61}$

Two characteristics were positively associated with tablet use among Veterans experiencing homelessness: a PTSD 
Table 4 Characteristics and Bivariate Comparisons (Unadjusted) Between Telehealth Tablet Users vs. Non-users Among Veterans Experiencing Homelessness, Stratified by Urban/Rural Location

\begin{tabular}{|c|c|c|c|c|c|c|}
\hline & $\begin{array}{l}\text { Urban homeless } \\
\text { tablet non-users } \\
N=615(\%)\end{array}$ & $\begin{array}{l}\text { Urban homeless } \\
\text { tablet users } \\
N=433(\%)\end{array}$ & $\begin{array}{l}P \text { - } \\
\text { value* }\end{array}$ & $\begin{array}{l}\text { Rural homeless } \\
\text { tablet non-users } \\
N=165(\%)\end{array}$ & $\begin{array}{l}\text { Rural homeless } \\
\text { tablet users } \\
N=232(\%)\end{array}$ & $\begin{array}{l}P \text { - } \\
\text { value* }\end{array}$ \\
\hline Age & & & $<0.001$ & & & $<0.001$ \\
\hline $18-44$ & $134(21.8)$ & $148(34.2)$ & & $48(29.1)$ & $89(38.4)$ & \\
\hline $45-64$ & $404(65.7)$ & $253(58.4)$ & & $87(52.7)$ & $129(55.6)$ & \\
\hline $65+$ & $77(12.5)$ & $32(7.4)$ & & $30(18.2)$ & $14(6.0)$ & \\
\hline Gender & & & 0.390 & & & 0.160 \\
\hline Female & $138(22.4)$ & $107(24.7)$ & & $25(15.2)$ & $48(20.7)$ & \\
\hline Male & 477 (77.6) & $326(75.3)$ & & $140(84.8)$ & $184(79.3)$ & \\
\hline Marital status & & & 0.004 & & & 0.700 \\
\hline Other & $506(84.5)$ & $327(77.3)$ & & $120(73.6)$ & $166(71.9)$ & \\
\hline Married & $93(15.5)$ & $96(22.7)$ & & $43(26.4)$ & $65(28.1)$ & \\
\hline Race/ethnicity & & & $<0.001$ & & & 0.260 \\
\hline White, non-Hispanic & $209(35.1)$ & $223(53.9)$ & & $121(74.2)$ & $169(73.8)$ & \\
\hline Black, non-Hispanic & $329(55.3)$ & $141(34.1)$ & & $23(14.1)$ & $27(11.8)$ & \\
\hline Hispanic & $28(4.7)$ & $28(6.8)$ & & $7(4.3)$ & $5(2.2)$ & \\
\hline Other & $29(4.9)$ & $22(5.3)$ & & $12(7.4)$ & $28(12.2)$ & \\
\hline $\begin{array}{l}\text { Homelessness after } 6 \\
\text { months }\end{array}$ & & & 0.003 & & & 0.047 \\
\hline No & $192(31.2)$ & $174(40.2)$ & & $68(41.2)$ & $119(51.3)$ & \\
\hline Yes & $423(68.8)$ & $259(59.8)$ & & $97(58.8)$ & $113(48.7)$ & \\
\hline Priority group & & & 0.005 & & & 0.260 \\
\hline $\begin{array}{l}\text { Group 1: service- } \\
\text { connected disabilities }\end{array}$ & $179(29.2)$ & $166(38.4)$ & & $56(34.1)$ & $87(37.7)$ & \\
\hline $\begin{array}{l}\text { Group 5: financial } \\
\text { insecurity }\end{array}$ & $256(41.8)$ & $148(34.3)$ & & $67(40.9)$ & $76(32.9)$ & \\
\hline Other than Groups 1 and 5 & $177(28.9)$ & $118(27.3)$ & & $41(25.0)$ & $68(29.4)$ & \\
\hline Chronic conditions (from & & & 0.140 & & & \\
\hline $\begin{array}{l}\text { list of 28) } \\
\text { Zero }\end{array}$ & $50(8.1)$ & 47 (10.9) & & $13(79)$ & $31(13.4)$ & 0.008 \\
\hline 1 to 2 & $220(35.8)$ & $167(38.6)$ & & $49(29.7)$ & $\begin{array}{l}31(13.4) \\
92(39.7)\end{array}$ & \\
\hline 3 or more & $345(56.1)$ & $219(50.6)$ & & $103(62.4)$ & $109(47.0)$ & \\
\hline MH diagnoses & $534(86.8)$ & 397 (91.7) & 0.013 & $150(90.9)$ & $215(92.7)$ & 0.520 \\
\hline Serious mental illness & $122(19.8)$ & $97(22.4)$ & 0.310 & $38(23.0)$ & $61(26.3)$ & 0.460 \\
\hline $\begin{array}{l}\text { Post-traumatic stress dis- } \\
\text { order }\end{array}$ & $269(43.7)$ & $247(57.0)$ & $<0.001$ & $78(47.3)$ & $136(58.6)$ & 0.025 \\
\hline Substance use disorder & $357(58.0)$ & $204(47.1)$ & $<0.001$ & $101(60.2)$ & $113(48.7)$ & 0.014 \\
\hline Depression & $433(70.4)$ & $304(70.2)$ & 0.940 & $118(71.5)$ & $167(72.0)$ & 0.920 \\
\hline Suicide risk flag & & & 0.760 & & & 0.780 \\
\hline No & $565(91.9)$ & $400(92.4)$ & & $145(87.9)$ & $206(88.8)$ & \\
\hline Yes & $50(8.1)$ & $33(7.6)$ & & $20(12.1)$ & $26(11.2)$ & \\
\hline
\end{tabular}

For homeless Veteran users and non-users: missing values exists in marital status (29), race/ethnicity (47), enrollment priority group (16), geographic location (25), drive time to VA secondary care (29)

For urban homeless Veteran users: missing values exists in marital status (26), racelethnicity (39), enrollment priority group (4)

For rural homeless Veteran users: missing values exists in marital status (3), racelethnicity (5), enrollment priority group (2), drive time to VA secondary care (4). *All P-values derive from chi-square tests comparing tablet users and non-users

diagnosis and residing in rural areas. Our finding that Veterans experiencing homelessness with PTSD demonstrated greater likelihood of tablet use is congruent with prior evidence of PTSD patients' acceptance and satisfaction with telemental health. ${ }^{31}$ A literature review of the adoption of telemental health for Veterans with PTSD found several facilitators: access to necessary electronic devices, availability of PTSDtrained clinicians, and supportive community. ${ }^{32}$ An examination of both video visits and My HealtheVet patient portal use among Veterans receiving VA mental health services found that Veterans with PTSD had substantially higher odds of video visit engagement $(\mathrm{AOR}=1.74,95 \% \mathrm{CI} 1.58-1.91)$ and being a dual user of both technologies $(\mathrm{AOR}=1.86$, 95\% CI 1.77-1.96) compared to Veterans without PTSD. ${ }^{55}$

The second factor positively associated with tablet use was rurality. Veterans experiencing homelessness residing in rural settings were more likely to use tablets than their urban counterparts (Table 4). This is consistent with recent research which showed that although rural Veterans had $17 \%$ lower odds of MHV patient portal use compared with urban patients, they were substantially more likely than their urban counterparts to engage in Clinical Video Telehealth or dual use of these resources. ${ }^{55}$ This may be because rural Veterans often live at a distance from VA medical centers, and that the cost of driving to in-person visits (e.g., in time, transportation, lost wages) can be considerable.

\section{Limitations}

Our study has a number of limitations. Our findings focused on Veterans within the VA system and on a single technology, so may not be generalizable to other populations and technologies. The tablet use examination period was short -6 months after receipt — so differences identified may have attenuated at 
Table 5 Telehealth Tablet Utilization, as Measured by VA Video Connect Visits-Comparisons (Unadjusted) Between Housed and Homeless Tablet Users, and Homeless Tablet Users Stratified by Urban/Rural Location

\begin{tabular}{|c|c|c|c|c|c|c|}
\hline & $\begin{array}{l}\text { Housed tablet users } \\
N=6133\end{array}$ & $\begin{array}{l}\text { Homeless tablet } \\
\text { users } N=675\end{array}$ & $\begin{array}{l}P \text { - } \\
\text { value }\end{array}$ & $\begin{array}{l}\text { Urban homeless tablet } \\
\text { users } N=433\end{array}$ & $\begin{array}{l}\text { Rural homeless tablet } \\
\text { users } N=\mathbf{2 3 2}\end{array}$ & $\begin{array}{l}P \text { - } \\
\text { value }\end{array}$ \\
\hline \multicolumn{7}{|l|}{ Mental health } \\
\hline $\begin{array}{l}\text { Mental health } \\
\text { visits, } N(\%)\end{array}$ & $4425(72.1)$ & $594(88.0)$ & $<0.001$ & $364(84.1)$ & $220(94.8)$ & $<0.001$ \\
\hline $\begin{array}{l}\text { Mean (SD) } \\
\text { Primary care }\end{array}$ & $3.6(4.4)$ & $3.9(4.3)$ & 0.098 & $3.8(4.4)$ & $4.0(4.1)$ & 0.49 \\
\hline $\begin{array}{l}\text { Primary care } \\
\text { Primary care visits, } \\
N(\%)\end{array}$ & $574(9.4)$ & $34(5.0)$ & $<0.001$ & $30(6.9)$ & $4(1.7)$ & 0.004 \\
\hline $\begin{array}{l}\text { Mean (SD)574 (9.4) } \\
\text { Any specialty care }\end{array}$ & $0.2(0.7)$ & $0.1(0.4)$ & 0.003 & $0.1(0.5)$ & $0(0.2)$ & 0.005 \\
\hline $\begin{array}{l}\text { Specialty care visits, } \\
N(\%)\end{array}$ & 1449 (23.6) & $81(12.0)$ & $<0.001$ & 59 (13.6) & $22(9.5)$ & 0.12 \\
\hline Mean (SD) & $1.0(2.8)$ & $0.3(1.1)$ & $<0.001$ & $0.3(1.1)$ & $0.3(1.2)$ & 0.67 \\
\hline
\end{tabular}

10 Veterans missing rural and urban information. *All P-values for continuous variables derive from t-test and P-values for categorical variables derived from chi-square tests. SD standard deviation

Table 6 Logistic Regression: Predicting Telehealth Tablet Use Among Veterans Experiencing Homelessness, and Stratified by Urban/Rural Location

\begin{tabular}{|c|c|c|c|c|c|c|}
\hline & \multicolumn{2}{|c|}{$\begin{array}{l}\text { All homeless tablet users }(N= \\
\text { 1395) }\end{array}$} & \multicolumn{2}{|c|}{$\begin{array}{l}\text { Urban homeless tablet users }(N \\
=1005)\end{array}$} & \multicolumn{2}{|c|}{$\begin{array}{l}\text { Rural homeless tablet users }(N= \\
\text { 390) }\end{array}$} \\
\hline & $\begin{array}{l}\text { Odds ratio }(95 \% \\
\text { CI) }\end{array}$ & $\begin{array}{l}P \text { - } \\
\text { value* }\end{array}$ & $\begin{array}{l}\text { Odds ratio }(95 \% \\
\text { CI) }\end{array}$ & $\begin{array}{l}P \text { - } \\
\text { value* }\end{array}$ & $\begin{array}{l}\text { Odds ratio }(95 \% \\
\text { CI) }\end{array}$ & $\begin{array}{l}P \text { - } \\
\text { value* }\end{array}$ \\
\hline \multicolumn{7}{|l|}{ Age } \\
\hline $18-44$ & $2.77(1.76,4.35)$ & $<0.001$ & $2.47(1.43,4.26)$ & 0.001 & $3.51(1.54,7.98)$ & 0.003 \\
\hline $45-64$ & $2.28(1.52,3.43)$ & $<0.001$ & $1.83(1.13,2.97)$ & 0.014 & $3.6(1.69,7.7)$ & 0.001 \\
\hline $65+$ & Reference & & Reference & & Reference & \\
\hline \multicolumn{7}{|l|}{ Gender } \\
\hline Male & Reference & & Reference & & Reference & \\
\hline Female & $0.95(0.72,1.27)$ & 0.736 & $0.94(0.67,1.31)$ & 0.714 & $1.08(0.6,1.94)$ & 0.800 \\
\hline \multicolumn{7}{|l|}{ Marital status } \\
\hline Married & $1.21(0.92,1.61)$ & 0.177 & $1.32(0.93,1.86)$ & 0.121 & $1.12(0.68,1.86)$ & 0.654 \\
\hline Other & Reference & & Reference & & Reference & \\
\hline \multicolumn{7}{|l|}{ Race/ethnicity } \\
\hline White, non-Hispanic & Reference & & Reference & & Reference & \\
\hline Black, non-Hispanic & $0.43(0.34,0.56)$ & $<0.001$ & $0.38(0.29,0.51)$ & 0.001 & $0.81(0.42,1.56)$ & 0.523 \\
\hline Hispanic & $0.74(0.44,1.24)$ & 0.252 & $0.81(0.45,1.44)$ & 0.471 & $0.36(0.1,1.23)$ & 0.104 \\
\hline Other & $0.89(0.56,1.42)$ & 0.628 & $0.62(0.34,1.15)$ & 0.129 & $1.45(0.67,3.12)$ & 0.347 \\
\hline \multicolumn{7}{|l|}{ Homelessness after 6 months } \\
\hline No & Reference & & Reference & & Reference & \\
\hline Yes & $0.75(0.59,0.96)$ & 0.023 & $0.81(0.61,1.09)$ & 0.166 & $0.64(0.4,1)$ & 0.052 \\
\hline \multicolumn{7}{|l|}{ Priority group } \\
\hline $\begin{array}{l}\text { Group 1: service-connected disabil- } \\
\text { ities }\end{array}$ & $1.03(0.76,1.39)$ & 0.861 & $1.04(0.72,1.5)$ & 0.839 & $1.02(0.58,1.8)$ & 0.949 \\
\hline Group 5: financial insecurity & Reference & & Reference & & Reference & \\
\hline Other than Groups 1 and 5 & $1.11(0.84,1.49)$ & 0.457 & $1.04(0.74,1.46)$ & 0.823 & $1.32(0.76,2.32)$ & 0.328 \\
\hline \multicolumn{7}{|l|}{ Urban/rural location } \\
\hline Urban & Reference & - & & & - & \\
\hline Rural* & $1.46(1.12,1.90)$ & 0.005 & - & & - & \\
\hline \multicolumn{7}{|l|}{ Chronic conditions (from list of 28) } \\
\hline Zero & Reference & & Reference & & Reference & \\
\hline 1 to 2 & $0.88(0.58,1.33)$ & 0.546 & $0.96(0.59,1.59)$ & 0.888 & $0.69(0.3,1.6)$ & 0.391 \\
\hline 3 or more & $0.76(0.5,1.16)$ & 0.206 & $0.94(0.57,1.56)$ & 0.821 & $0.44(0.19,1.03)$ & 0.059 \\
\hline \multicolumn{7}{|l|}{ Depression } \\
\hline Yes & $1.02(0.79,1.33)$ & 0.866 & $1.02(0.75,1.38)$ & 0.923 & $1.17(0.69,1.99)$ & 0.568 \\
\hline No & Reference & & Reference & & Reference & \\
\hline \multicolumn{7}{|l|}{ Post-traumatic stress disorder } \\
\hline Yes & $1.64(1.27,2.12)$ & $<0.001$ & $1.69(1.25,2.29)$ & 0.001 & $1.54(0.95,2.48)$ & 0.080 \\
\hline No & Reference & & Reference & & Reference & \\
\hline \multicolumn{7}{|l|}{ Substance use disorder } \\
\hline Yes & $0.59(0.46,0.76)$ & $<0.001$ & $0.58(0.43,0.78)$ & 0.001 & $0.6(0.37,0.96)$ & 0.032 \\
\hline No & Reference & & Reference & & Reference & \\
\hline \multicolumn{7}{|l|}{ Serious mental illness } \\
\hline Yes & $1.20(0.91,1.58)$ & 0.208 & $1.17(0.84,1.63)$ & 0.358 & $1.36(0.8,2.3)$ & 0.251 \\
\hline No & Reference & & Reference & & Reference & \\
\hline
\end{tabular}

*All P-values derive from logistic regression 
12 months after receipt. As a cross-sectional study with diagnoses identified in the 12 months prior to tablet receipt, some conditions may have resolved (e.g., depression) prior to the start of the use, thus leading to misclassification bias. The indicator of homelessness in this study was broad; e.g., it did not differentiate between chronic and temporary homelessness. Examining such differences was beyond the scope of this study. The reliability of the data indicating patients' current homeless status may be hindered by the fact that Veterans experiencing homelessness may move frequently; hence, the electronic health record may not reflect the most recent residence. Future studies should examine whether different types of homelessness are differentially associated with technology adoption and use.

\section{CONCLUSIONS}

In providing hardware and wireless telehealth access, VA's tablet distribution program is a promising model to help vulnerable individuals receive virtual care. But supportive structures and interventions may be needed (and are the focus of an ongoing qualitative study by the authors) to strengthen its success through training for digital literacy, accessibility for those with physical or other impairments, and dissemination of information to both patients and providers. Target groups among patients experiencing homelessness who may need more tablet assistance include those who are older, Black, or with a SUD. In general, while living in rural areas appeared to boost the use of video visits, rural patients facing multiple chronic conditions or access disadvantages would benefit from additional assistance in their adoption and use. Telehealth for vulnerable populations has become particularly salient during the COVID-19 pandemic. Yet without support for marginalized populations to access telehealth, the pandemic or digital divide may further widen the gulf between those with and without access to healthcare.

Corresponding Author: Lynn A. Garvin, PhD, MBA; VA Center for Healthcare Organization and Implementation Research (CHOIR), VA Boston Healthcare System, 150 S. Huntington Avenue, Bldg 9, Rm 225, Boston, MA 02130, USA (e-mail: Lynn.Garvin@va.gov).

Funding Dr. Garvin's and Dr. McInnes' work was funded by the US Department of Veterans Affairs (VA) National Center for Homelessness Among Veterans (Garvin, XVA 11-056). Dr. Zulman's, Ms. Hu's, and Ms. Slightam's work was funded by the VA Health Services Research \& Development/Quality Enhancement Research Initiative (HSR\&D/ QUERI) (Zulman, PEI 18-205). The views expressed in this manuscript are those of the authors and do not necessarily reflect the views of the US Department of Veterans Affairs or the US government.

\section{Declarations:}

Conflict of Interest: The authors declare that they do not have a conflict of interest.

\section{REFERENCES}

1. Darkins A, Foster L, Anderson C, Goldschmidt L, Selvin G. The design, implementation, and operational management of a comprehensive quality management program to support national telehealth networks. Telemedicine and e-Health. 2013;19(7):557-564.

2. Adams SV, Mader MJ, Bollinger MJ, Wong ES, Hudson TJ, Littman AJ. Utilization of Interactive Clinical Video Telemedicine by Rural and Urban Veterans in the Veterans Health Administration Health Care System. Journal of Rural Health. 2019;35(3):308-318.

3. Chen CK, Palfrey A, Shreck E, et al. Implementation of Telemental Health (TMH) psychological services for rural veterans at the VA New York Harbor Healthcare System. Psychological services. 2019;

4. Brearly TW, Goodman CS, Haynes C, McDermott K, Rowland JA. Improvement of postinpatient psychiatric follow-up for veterans using telehealth. American Journal of Health-System Pharmacy. 2020;77(4):288-294.

5. Shreck E, Nehrig N, Schneider JA, et al. Barriers and facilitators to implementing a US Department of Veterans Affairs Telemental Health (TMH) program for rural veterans. Journal of Rural Mental Health. 2020;41(1): 1 .

6. Heyworth L, Corrigan K, Schectman G. Video Visits in Primary Care: A Nationwide Pilot Study at the Veterans Health Administration. Springer 233 Spring St, New York, NY 10013 USA; 2017:S807-S808.

7. Ambert-Pompey S, Konecky B, Ahlstrom D. Improving Access: Teambased Primary Care via Telehealth in the VA. SGIM Forum. 2017;40(10): 13.

8. Eberly LA, Kallan MJ, Julien HM, et al. Patient characteristics associated with telemedicine access for primary and specialty ambulatory care during the COVID-19 pandemic. JAMA network open. 2020;3(12):e2031640-e2031640.

9. Jordan DN, Jessen CM, Ferucci ED. Views of Patients and Providers on the Use of Telemedicine for Chronic Disease Specialty Care in the Alaska Native Population. Telemedicine and e-Health. 2020;

10. Lesher AP, Fakhry SM, DuBose-Morris R, et al. Development and evolution of a statewide outpatient consultation service: leveraging telemedicine to improve access to specialty care. Population health management. 2020;23(1):20-28.

11. Lum HD, Nearing K, Pimentel CB, Levy CR, Hung WW. Anywhere to Anywhere: Use of Telehealth to Increase Health Care Access for Older, Rural Veterans. Public Policy \& Aging Report. 2020;30(1):12-18.

12. Shura RD, Brearly TW, Tupler LA. Telehealth in Response to the COVID19 Pandemic in Rural Veteran and Military Beneficiaries. The Journal of Rural Health. 2021;37:200-204.

13. Padala KP, Wilson KB, Gauss CH, Stovall JD, Padala PR. VA video connect for clinical care in older adults in a rural state during the COVID-19 pandemic: cross-sectional study. J Med Internet Res. 2020;22(9):e21561.

14. Byrne T, Cusack M, True G, Montgomery AE, Smith M. "You Don't See Them on the Streets of Your Town": Challenges and Strategies for Serving Unstably Housed Veterans in Rural Areas. Housing Policy Debate. 2020;30(3):409-430.

15. Cotner B, Ottomanelli L, O'Connor DR, Njoh E, Jones V. Delivering vocational services to rural veterans with disabilities through video telerehabilitation. Archives of Physical Medicine and Rehabilitation. 2016;97(10):e95.

16. Glynn LH, Chen JA, Dawson TC, Gelman H, Zeliadt SB. Bringing chronic-pain care to rural veterans: A telehealth pilot program description. Psychological Services. 2020;

17. Cheng MK, Allison TA, McSteen BW, Cattle CJ, Lo DT. The Adoption of Video Visits During the COVID-19 Pandemic by VA Home Based Primary Care. Journal of the American Geriatrics Society. 2020;

18. Kruse C, Fohn J, Wilson N, Patlan EN, Zipp S, Mileski M. Utilization barriers and medical outcomes Commensurate with the use of telehealth among older adults: systematic review. JMIR medical informatics. 2020;8(8):e20359.

19. Moo LR, Gately ME, Jafri Z, Shirk SD. Home-based video telemedicine for dementia management. Clinical Gerontologist. 2020;43(2):193-203.

20. Ali D, Gillie M, Carstarphen KJ. Bridging the digital divide for vulnerable patient telehealth. Innovation in Aging. 2020;4(Suppl 1):928.

21. Parker S, Prince A, Thomas L, Song H, Milosevic D, Harris MF. Electronic, mobile and telehealth tools for vulnerable patients with chronic disease: a systematic review and realist synthesis. BMJ Open. 2018;8(8):e019192. 
22. Pekmezaris R, Kozikowski A, Pascarelli B, et al. A Telehealth-Delivered Pulmonary Rehabilitation Intervention in Underserved Hispanic and African American Patients With Chronic Obstructive Pulmonary Disease: A Community-Based Participatory Research Approach. JMIR Formative Research. 2020;4(1):e13197.

23. King DA KS, Polo J, Solomon J, Pekmezaris R, Hajizadeh N. . Optimizing Telehealth Experience Design Through Usability Testing in Hispanic American and African American Patient Populations: Observational Study JMIR Rehabil Assist Technol. 2020;7(2):e16004.

24. Murry VM, Berkel C, Inniss-Thompson MN, Debreaux ML. Pathways for African American success: Results of three-arm randomized trial to test the effects of technology-based delivery for rural African American families. Journal of pediatric psychology. 2019;44(3):375-387.

25. McCall T, Iii B, McCall R, Khairat S. The Use of Culturally-Tailored Telehealth Interventions in Managing Anxiety and Depression in African American Adults: A Systematic Review. Studies in health technology and informatics. 2019;264:1728-1729.

26. Kohn LL, Introcaso CE. A Cultural Context for Providing Dermatologic Care to American Indian and Alaskan Native Communities Through Telehealth. JAMA Dermatology. 2019;155(8):884-886.

27. Morenz AM, Wescott S, Mostaghimi A, Sequist TD, Tobey M. Evaluation of barriers to telehealth programs and dermatological care for American Indian individuals in rural communities. JAMA Dermatology. 2019;155(8):899-905.

28. Myers US, Coulon S, Knies K, et al. Lessons learned in implementing VA video connect for evidence-based psychotherapies for anxiety and depression in the Veterans Healthcare Administration. Journal of tech nology in behavioral science. 2020:1-7.

29. Ecker $\mathrm{AH}$, Amspoker AB, Hogan JB, Lindsay JA. The Impact of Cooccurring Anxiety and Alcohol Use Disorders on Video Telehealth Utilization Among Rural Veterans. Journal of technology in behavioral science. 2020:1-6.

30. Kruse CS, Lee K, Watson JB, Lobo LG, Stoppelmoor AG, Oyibo SE. Measures of effectiveness, efficiency, and quality of telemedicine in the management of alcohol abuse, addiction, and rehabilitation: systematic review. J Med Internet Res. 2020;22(1):e13252.

31. Turgoose D, Ashwick R, Murphy D. Systematic review of lessons learned from delivering tele-therapy to veterans with post-traumatic stress disorder. Journal of telemedicine and telecare. 2018;24(9):575-585.

32. Kruse CS, Atkins JM, Baker TD, Gonzales EN, Paul JL, Brooks M. Factors influencing the adoption of telemedicine for treatment of military veterans with post-traumatic stress disorder. Journal of rehabilitation medicine. 2018;50(5):385-392.

33. Lin LA, Casteel D, Shigekawa E, Weyrich MS, Roby DH, McMenamin SB Telemedicine-delivered treatment interventions for substance use disorders: A systematic review. J Subst Abuse Treat. 2019;101:38-49.

34. Lin L, Fernandez AC, Bonar EE. Telehealth for Substance-Using Populations in the Age of Coronavirus Disease 2019: Recommendations to Enhance Adoption. JAMA Psychiatry. 2020;77(12):1209-1210. doi:https://doi.org/10.1001/jamapsychiatry.2020.1698

35. Brunet N, Moore, D. T., Lendvai Wischik, D., Mattocks, K. M., \& Rosen, M. I. Increasing buprenorphine access for veterans with opioid use disorder in rural clinics using telemedicine. Substance Abuse. 2020:1-8.

36. Basit SA, Mathews N, Kunik ME. Telemedicine interventions for medication adherence in mental illness: A systematic review. General hospital psychiatry. 2020;62:28-36.

37. Henry M, Watt R, Mahathey A, Ouellette J, Sitler A. The 2019 Annual Homeless Assessment Report (AHAR) to Congress, PART 1: Point-in-Time Estimates of Homelessness. Washington, DC: Office of Community Planning and Development. US Dept of Housing and Urban Development, January 2020. Available at: https://files.hudexchange.info/resources/ documents/2019-AHAR-Part-1.pdf Accessed June 1, 2020.

38. Tsai J, Snitkin M, Trevisan L, Kraus SW, Pietrzak RH. Awareness of suicide prevention programs among US military veterans. Administration and Policy in Mental Health and Mental Health Services Research. 2020;47(1):115-125.

39. McInnes DK, Cutrona SL. Homeless Veterans and Use of Information Technology. Homelessness Among US Veterans: Critical Perspectives. 2018:245.

40. Miller CJ, McInnes DK, Stolzmann K, Bauer MS. Interest in use of technology for healthcare among veterans receiving treatment for mental health. Telemedicine and e-Health. 2016;22(10):847-854
41. Zulman DM, O’Brien C, Slightam C, Nevedal A, Breland JY. Strategies to engage high-risk, complex patients in intensive outpatient care programs. $J$ Gen Intern Med. 2016;31:S405-S405.

42. Zulman DM, Chang ET, Wong A, et al. Effects of intensive primary care on high-need patient experiences: Survey findings from a Veterans Affairs randomized quality improvement trial. J Gen Intern Med. 2019:1-7.

43. Zulman DM, Wong EP, Slightam C, et al. Making connections: nationwide implementation of video telehealth tablets to address access barriers in veterans. JAMIA Open. 2019;2(3):323-329.

44. Jacobs JC, Hu J, Slightam C, Gregory A, Zulman DM. Virtual Savings: Patient-Reported Time and Money Savings from a VA National Telehealth Tablet Initiative. Telemedicine and e-Health. 2019;

45. Belkora J, Volz S, Loth M, et al. Coaching patients in the use of decision and communication aids: RE-AIM evaluation of a patient support program. BMC Health Serv Res. 2015;15(1):209.

46. Glasgow RE, Vogt TM, Boles SM. Evaluating the public health impact of health promotion interventions: the RE-AIM framework. American journal of public health. 1999;89(9):1322-1327.

47. US Dept of Veterans Affairs, Office of Rural Health, Definition of Rurality. Available at: https://www.ruralhealth.va.gov/rural-definition.asp.

48. Montgomery AE, Szymkowiak D, Tsai J. Housing Instability and Homeless Program Use Among Veterans: The Intersection of Race, Sex, and Homelessness. Housing Policy Debate. 2020;30(3):396-408.

49. Centers for Disease Control and Prevention, National Center for Health Statistics. https://www.cdc.gov/nchs/icd/icd9.htm [Accessed on: March 14, 2021].

50. US Department of Veterans Affairs, Managerial Cost Accounting Office, Program Documents - FY21 Mid-Year Active Stop Codes. http://vaww. dss.med.va.gov/programdocs/pd_oident.asp [Accessed on: March 14, 2021].

51. Wildenbos GA, Maasri K, Jaspers M, Peute L. Older adults using a patient portal: registration and experiences, one year after implementation. Digital health. 2018;4:2055207618797883.

52. Understanding PTSD and Aging. Washington, DC: National Center for PTSD, US Dept of Veterans Affairs. Published September 2019. https:// www.ptsd.va.gov/publications/print/understandingptsd_aging_booklet. pdf [].

53. Currie M, Philip LJ, Roberts A. Attitudes towards the use and acceptance of eHealth technologies: a case study of older adults living with chronic pain and implications for rural healthcare. BMC Health Serv Res. 2015;15(1): 162 .

54. Connolly SL, Miller CJ, Koenig CJ, et al. Veterans' attitudes toward smartphone App use for mental health care: qualitative study of rurality and age differences. JMIR mHealth and uHealth. 2018;6(8):e10748.

55. Abel EA, Shimada SL, Wang K, et al. Dual use of a patient portal and clinical video telehealth by veterans with mental health diagnoses: retrospective, cross-sectional analysis. J Med Internet Res. 2018;20(11):e11350.

56. Eliacin J, Matthias MS, Cunningham B, Burgess DJ. Veterans' perceptions of racial bias in VA mental healthcare and their impacts on patient engagement and patient-provider communication. Patient Educ Couns. 2020;103(9):1798-1804

57. Milam AJ, Furr-Holden D, Edwards-Johnson J, et al. Are clinicians contributing to excess African American COVID-19 deaths? Unbeknownst to them, they may be. Health Equity. 2020;4(1):139-141.

58. Sewell AA. Disaggregating ethnoracial disparities in physician trust. Social Science Research. 2015;54:1-20.

59. Gaudiano B, Moitra E. Leveraging Mobile Technology to Improve the Treatment of Comorbid Bipolar and Substance Use Disorders. J Addict Res Ther. 2015;6:e126.

60. Kazemi DM, Borsari B, Levine MJ, Li S, Lamberson KA, Matta LA. A systematic review of the mHealth interventions to prevent alcohol and substance abuse. J Health Commun. 2017;22(5):413-432.

61. Slightam C, Gregory AJ, Hu J, et al. Patient Perceptions of Video Visits Using Veterans Affairs Telehealth Tablets: Survey Study. J Med Internet Res. 2020;22(4):e15682.

Publisher's Note Springer Nature remains neutral with regard to jurisdictional claims in published maps and institutional affiliations. 